\section{Listeria infections in the newborn}

Sir,

Robertson et al. ${ }^{1}$ appear to be right in thinking that neonatal listeriosis is under reported. In the UK cases have been recorded as follows: 1977, 11 babies (including 4 deaths); 1978, 19 babies (including 6 deaths); and in the first 9 months of 1979, 11 babies (including 2 deaths) (Public Health Laboratory Service, unpublished data). Reporting of this infection to the PHLS is voluntary and these figures must represent only a proportion of the actual number of cases.

Neonatal listeriosis carries a high mortality, but the illness can be relatively slight, as exemplified by 2 babies born in July 1979 at Leamington Spa.

The first, a girl, was born by normal vaginal delivery after spontaneous premature labour at 33 weeks' gestation. Her mother developed a pyrexia of $38.5^{\circ} \mathrm{C}$ during labour. The membranes ruptured 10 minutes before delivery. The liquor was meconium-stained. The baby, weighing $2.0 \mathrm{~kg}$, had grunting respiration for the first hour after birth and recurrent apnoea, responsive to stimulation, for 12 hours. Her temperature reached $38^{\circ} \mathrm{C}$. Blood (from which Listeria monocytogenes was subsequently isolated) was taken one hour after birth and she was started on parenteral ampicillin and made a prompt recovery. CSF was not examined. Maternal blood and vaginal swab showed no pathogens.

The second baby, also a girl, was born 3 days after the first by emergency caesarean section for failure to progress. Labour had been induced at 38 weeks with vaginal prostaglandin because of raised maternal blood pressure and low urinary oestriols. The membranes ruptured 10 hours before birth. The baby, weighing $2 \cdot 30 \mathrm{~kg}$, was well for the first 72 hours and then developed diarrhoea and a temperature of $39.5^{\circ} \mathrm{C}$. L. monocytogenes was isolated from blood culture. CSF contained no cells and was sterile. No pathogens were isolated from faeces, urine, or pharyngeal aspirate, nor were they isolated from maternal vaginal swab. The baby was treated with penicillin and gentamicin, and then with ampicillin as soon as the results of the cultures were known. Apart from a transient rash, she made an uneventful recovery.

The fact that the first baby's blood culture was positive so soon after birth, makes it likely that the infection was acquired in utero despite the negative maternal cultures. The second baby is assumed to have acquired the infection after birth by cross-infection either in the labour room or in the nursery. No other baby in the nursery was affected.

Congenital listeriosis most commonly occurs in preterm infants, perhaps because the maternal illness initiates premature labour. In addition, preterm babies are more susceptible than term ones to acquiring infection after birth. Cross-infection in a neonatal nursery is a potential hazard and affected babies should be treated in isolation.

\section{Reference}

Robertson M H, Mussalli N G, Aizad T A, Okaro J M, Banwell G S. Two cases of perinatal listeriosis. Arch Dis Child 1979; 54: 549-62.

C N Storrs and J W Partridge Department of Paediatrics, Warwick Hospital, Lakin Road, Warwick CV34 5BW 